\title{
Dynamic Forecasting Algorithm of Inbound Ice and Snow Tourism in China Based on Improved Deep Confidence Network
}

\author{
Yuguang Zhao, ${ }^{1}$ Chuanming Jiao $\mathbb{D}^{0},{ }^{2}$ Jinhui Li, ${ }^{3}$ Zhigang Yuan, ${ }^{4}$ Xin $\mathrm{Li}^{2}{ }^{2}$ Hanghai Gu, ${ }^{5}$ \\ and Zhong Zhang ${ }^{2}$ \\ ${ }^{1}$ School of Public Health, Qiqihar Medical University, Qiqihar, Heilongiiang 161006, China \\ ${ }^{2}$ Physical Education Department, Qiqihar Medical University, Qiqihar, Heilongjiang 161006, China \\ ${ }^{3}$ Physical Education College, Qiqihar University, Qiqihar, Heilongjiang 161006, China \\ ${ }^{4}$ Harbin Institute Physical Education, Harbin, Heilongjiang 150001, China \\ ${ }^{5}$ Qiqihar Federation of Literary and Art Circles, Qiqihar, Heilongjiang 161000, China
}

Correspondence should be addressed to Chuanming Jiao; jiaochuanming@qmu.edu.cn

Received 19 August 2021; Revised 30 September 2021; Accepted 4 October 2021; Published 25 October 2021

Academic Editor: Fazlullah Khan

Copyright (c) 2021 Yuguang Zhao et al. This is an open access article distributed under the Creative Commons Attribution License, which permits unrestricted use, distribution, and reproduction in any medium, provided the original work is properly cited.

\begin{abstract}
Ice and snow-based tourism is getting popular around the world and it is one of the major sources of revenue for a region with required facilities. According to a report by China Daily, China was expected to witness 230 million tourist visits in 2020-2021 with a total revenue generation surpassing 390 billion yuan. In order to promote the ice and snow tourism, proper arrangements such as accommodation, transport facility, and energy provision for heating and food need to be arranged as per the demand of the visitors at a certain period of time. A tourist prediction system can help in this regard for good estimation but considering the problems of traditional ice and snow tourism systems, specifically the prediction accuracy and long forecasting time, a dynamic forecasting algorithm for ice and snow inbound tourism based on an improved deep confidence network is proposed. The system analyzes the relationship between the demand for ice and snow inbound tourism and the level of national economic development, people's living standards, demographic characteristics, traffic conditions, and tourism supply capacity. It then takes the influencing factors of ice and snow inbound tourism demand as sample data and arranges the sample data sequence. The inbound tourism demand dynamic prediction model uses an improved deep confidence network to learn and train the prediction model, input test data into the trained model, and output the dynamic prediction value of ice and snow inbound tourism demand in the output layer to obtain the prediction result. The simulation results show that the proposed algorithm has improved accuracy in predicting the demand of inbound tourism for ice and snow, whereas the forecasting time is reduced.
\end{abstract}

\section{Introduction}

It has certain reference significance for other cyclical tourism forecast researches. Judging from the changing trend of inbound tourism demand for ice and snow, it is in an upward cycle.

The number of tourists is the basic indicator of the development of tourism, and whether the source of tourists is sufficient is the key to the development of the entire tourism industry. Moreover, the tourism industry can only grow if proper proactive planning is carried out by the concerned departments and sufficient resources are available for the tourists at their desired locations. It should be kept in mind that a happy tourist can pull multiple other tourists in an area which is key to the success of the tourism market. As such, the scientific forecast of the tourist source market can provide a reliable basis for formulating tourism development strategies and tourism planning and development. In modern tourism research, forecasting the tourism market is a difficult area [1] that involves multidisciplinary study. Apart from other forms of tourism, ice and snow tourism is showing a diversified and high-level development trend and as one of the most distinctive types of tourism, it has become one of the fastest growing parts of the entire tourism industry and enjoys a high reputation specifically in China. Yet in international perspective, the influence of inbound ice and snow tourism in China lags far 
behind that of Europe and North America where ice and snow tourism started earlier and is promoted strategically [2]. Compared with production industries such as industry and agriculture, the tourism industry of ice and snow is characterized by its volatility because it is influenced by a number of factors including income level, leisure time, age structure, education level, occupation, family structure, and other market forces of the residents in the source market. Moreover, factors such as gross national product, technological progress, taxation, natural disasters, war, terrorism, level of urbanization, other large-scale festivals, and other social and economic elements also affect the ice and snow tourism significantly. Therefore, it is difficult to precisely calculate the macroforecast and microforecast of the demand for ice and snow inbound tourism. As such, incorporating modern technologies that utilize artificial intelligence for the development of a scientific and operational dynamic forecasting model for ice and snow tourism can effectively solve the problem [3]. Considering the global development trend, the system can be used for targeted, planned, and scientific publicity and promotion that could be carried out for the international tourist source market of ice and snow tourism. Furthermore, the ice and snow tourism resources could be integrated with neighboring provinces; northern folklore and other regional cultural characteristics can be used to enhance the overall tourist experience and competitiveness. This could be spread across international boundaries by strengthening cooperation with foreign ice and snow industries in order to learn advanced business concepts as well as common management standards and systems for the world's ice and snow industries.

Although tourism demand forecasting by utilizing scientific methods is a relatively new research area, researchers have proposed some models targeted towards the said problem. However, with the passage of time and new developments, these forecasting methods are becoming more complex and diversified. In a related study conducted by Yao et al., a system based on an improved grey model is proposed [4]. In this study, the demand forecasting algorithm firstly determines the optimal number of input subsets by the input subset method according to the average absolute error percentage of the first-order Grey Model GM $(1,1)$ forecast and then uses the fuzzy set theory to take the calculated membership vector. In the end, the weight of the Markov transition matrix vector is used to optimize the predicted value. In order to be able to predict according to the passage of time, a dynamic prediction model with equal dimensions and recursive compensation is established. In a related study, Mingyang et. al. [5] proposed an improved Drosophila algorithm to optimize the travel demand prediction algorithm of the echo state network. The standard Drosophila optimization algorithm known as Fruit Fly Optimization Algorithm (FOA) is a recently proposed swarm intelligence-based method which incorporates fruit fly foraging behavior to solve global optimization method. Although it has certain advantages like simple structure to be realized with computer and ease of understanding, it has certain limitations including low convergence precision and getting easily trapped in a local optimum value at the later evolution stage. Thus, an improvement is proposed in which the number of Drosophila population and search steps were adjusted adaptively. At the same time, the initial iteration position was optimized to improve the algorithm. Local search capability and search efficiency were also tuned before combining the improved FOA algorithm, AFOA, with the echo state network (ESN) to construct a two-stage combined prediction model (AFOAESN). This method initially obtains its key parameters through the AFOA optimization and then inputs the optimized parameters into the ESN to form the final combined forecasting model. In the end, this model is utilized to forecast tourism demand. However, the prediction accuracy of the above two algorithms for tourism demand forecasting is relatively low, resulting in poor forecasting effect and making them ineffective choice for practical use.

Apart from Yao et al. [4] and Xu [5], in an earlier study, Liang et. al. [6] proposed an autoregressive distributed lag model prediction algorithm for tourism demand based on searching big data to build an autoregressive distributed lag model (ARDL) to predict tourism demand. The research content in this study is based on the United States where an autoregressive distribution lagging model is established and predictive analysis is performed. Similarly, Han and Fang proposed a tourism demand forecasting algorithm based on the GIOWHA-GALSSVR-SARIMA combined model [7]. Firstly, the Genetic Algorithm-Least Squares Support Vector Regression (GA-LSSVR) was constructed with the lagging values of three indicators: urban residents' per capita disposable income, consumer expectations index, and the number of overnight visitors as input variables. The model and the seasonally adjusted Autoregressive Integrated Moving Average (ARIMA) model preliminarily predicted the number of overnight tourists in the study. Then, a combination model based on the GIOWHA operator is established to complete the tourism demand forecast. However, the above two algorithms take a long time to predict tourism demand, which leads to low efficiency of these prediction systems.

Aiming at the problems of the above algorithms, this paper proposes a dynamic forecasting algorithm for inbound tourism demand based on an improved deep belief network and verifies the effectiveness of this algorithm through simulation experiments. It solves the problems in the traditional algorithm and lays a foundation for the development of inbound ice and snow-based tourism that could be adopted for practical applications.

\section{Analysis on the Influencing Factors of the Demand for Ice and Snow Inbound Tourism}

Ice and snow inbound tourism has grown gradually in the past ten years. Researchers have identified multiple factors that can affect the number of visiting tourists and the overall tourism in an area. The primary condition for the growth of tourism is the increase in people's freely disposable income. In addition, there are also a series of social factors related to ice and snow inbound tourism, such as the level of national economic development, demographic characteristics, traffic conditions, and tourism supply capacity. These conditions contribute to the overall demand for ice and snow inbound 
tourism. This paper considers the amount of ice and snow inbound tourism demand as the target quantity and studies its relationship with the national economic development level, people's living standards, demographic characteristics, traffic conditions, and tourism supply capacity for a better forecasting purpose.

\subsection{The Relationship between the Demand for Ice and Snow} Inbound Tourism and the Level of National Economic Development. The demand for ice and snow inbound tourism, in general, refers to the number of tourists who are visiting an area and are able to purchase tourism products with a certain currency payment capacity in a specific period of time. The level of national economic development contributes significantly to the demand for ice and snow inbound tourism. As the national economy of a country develops, the investment in infrastructure construction projects will inevitably increase. The construction of transportation infrastructure, the expansion of hotels and hostels, and the infrastructure construction primarily for tourist attractions will greatly stimulate the growth of tourism. On the other hand, the increase in demand for inbound tourism plays an important role in promoting the development of the national economy, and so the two complement each other. For the current study, the Gross Domestic Product (GDP) is selected to reflect the level of development of the national economy [8].

\subsection{The Relationship between the Demand for Ice and Snow} Inbound Tourism and People's Living Standards. In this study, people's living standards are reflected by residents' disposable income. Disposable income refers to the remaining balance after deducting all taxes and social consumption from the personal income that people earn by getting engaged in different social and economic activities, as well as the part that must be consumed in daily life. It is the part of the income that people can arbitrarily decide on their use. When people's necessities like food, clothing, shelter, and transportations are fulfilled, people can have higher life pursuits and tourism is one of them. Therefore, people's living standards are one of the main factors affecting the demand for ice and snow inbound tourism. The difference in tourists' discretionary income will affect tourists' travel mode, accommodation conditions, dining conditions, and choice of tourist destinations. Therefore, the amount of discretionary income is closely related to the demand for ice and snow inbound tourism [9].

\subsection{The Relationship between the Demand for Ice and Snow} Inbound Tourism and Demographic Characteristics. The expansion of the population will increase the demand for ice and snow inbound tourism. Although this effect is observable in years of time, the demand for ice and snow inbound tourism is closely related to the demographic characteristics of the tourist source area. The different age compositions, different sex ratios, and total population of the tourist source areas will have different effects on the demand of tourism. In addition, the economic development level of the tourist source area also affects the population's difference in the demand of tourism. If the tourist source area is economically developed, the population growth will greatly lead to the increase in tourism demand, while on the contrary, if the tourist source area is relatively backward economically, the contribution of population growth to tourism demand will be relatively small [10].

\subsection{The Relationship between Ice and Snow Inbound Tourism} Demand and Traffic Conditions. Traffic conditions have a crucial impact on the demand of tourism especially ice and snow inbound tourism. One of the primary issues for tourists while traveling abroad is the transportation problem. With the rapid development of China's economy, China's roads, railways, aviation, and other basic transportation facilities have become more and more accommodating, and tourists have more and more choices of transportation options for traveling. The improvements like rapid construction of highways, the operation of high-speed trains, and the development of air transportation have greatly shortened the time for tourists to reach tourist destinations, so that they can spend more time on tourism [11]. Moreover, as the facilities of transportation are upgraded, tourists feel more comfortable and safer during the journey, feel less fatigue of the journey, and can better enjoy the trip. The current study selects the traffic mileage of the transportation line as a parameter to reflect the traffic conditions for research purposes.

\subsection{The Relationship between the Demand for Ice and Snow} Inbound Tourism and the Capacity of Tourism Supply. The supply of different commodities to the target tourism area is also a key parameter to consider while promoting tourism in a region. This involves different types of products provided to the tourism market at a certain price within a certain period of time, including different types of gears required for specific tourism, for example, ice and snowbased tourism, tourist facilities, and tourism services provided by the operators. The current study primarily selects ice and snow inbound tourism facilities as an indicator of tourism supply capacity. Tourist facilities are used by tourism operators to provide tourists with food, accommodation, transportation, travel, shopping, entertainment, and other related facilities to directly develop tourism activities. They are the dominant part of the investment of ice and snow inbound tourism enterprises. The tourism supply represents a rigid indicator of local reception capacity. The reception capacity reflects the strength and development scale of a country or region to accommodate incoming people. Generally speaking, the ice and snow inbound tourist facilities mainly include four parts: tourist transportation, tourist accommodation and reception facilities, tourist entertainment facilities, and tourist shopping facilities. This current study focuses on the number of travel agencies and star-rated hotels to reflect the supply capacity of ice and snow inbound tourism. 


\section{Construction of a Dynamic Forecasting Model for the Inbound Tourism Demand of Ice and Snow}

Based on the aforementioned analysis of the relationships between the demand for ice and snow inbound tourism and the level of national economic development, people's living standards, demographic characteristics, traffic conditions, and tourism supply capacity, a fuzzy time series is used to construct a dynamic forecast model for inbound tourism demand for ice and snow. The use of fuzzy sets for modeling and predicting time series arises almost intuitively. The idea is to divide the universe of discourse from time series in fuzzy set's intervals and then learn how each area behaves by extracting the rules via time-series patterns. The fuzzy models not only assist in the formation of approximate functions but also enhance the readability of rules using linguistic variables that make them more accessible for profound analysis. For clarification, the fuzzy time series is comprised of four steps:

(1) Defining the universe and divide the interval

(2) Incorporation of fuzzy historical data

(3) Establishing fuzzy logic relationships and obtain fuzzy relationship groups

(4) Defuzzify relationships and build predictive models [12]

Step 1: data adjustment: in the first step, the relationship between the demand for ice and snow inbound tourism and the influencing factors including the level of national economic development, people's living standards, demographic characteristics, traffic conditions, and tourism supply capacity is taken as sample data which is arranged and processed accordingly. For simplicity, the data sequence is denoted as $d_{1}, d_{2}, d_{3}, \ldots, d_{n}$. Step 2: define the universe of observations and divide the interval.

In order to process the data sequence, find the minimum $d_{1}$ and maximum $d_{n}$ in the sequence.
The universe $U$ can then be defined as denoted in the following equation:

$$
U=\left[d_{1}-\sigma_{1}, d_{n}-\sigma_{2}\right],
$$

where $\sigma_{1}, \sigma_{2}$ represent two suitable positive numbers.

To simplify the procedure, Statistical Package for Social Sciences (SPSS) 20.0, a software package specifically designed by IBM for interactive statistical analysis, is used to call the $k$-means cluster process to perform cluster analysis on the $d_{1}, d_{2}, d_{3}, \ldots, d_{n}$ sequence to obtain $k$ cluster centers, then calculate the median values of the $k$ centers adjacent to each other to obtain $k-1$ values, and put them into the universe $U$. In $U, k$ initial intervals are obtained which are denoted as $u_{1}, u_{2}, u_{3}, \ldots, u_{k}$. Initially, the absolute values of the difference between the two adjacent data in the sequence $d_{1}, d_{2}, d_{3}, \ldots, d_{n}$ are calculated followed by the average of all the absolute values. The half of the average of the absolute values is regarded as the maximum allowable distance of the interval, and then the values are, respectively, put into $u_{1}, u_{2}, u_{3}, \ldots, u_{k}$, and the interval is divided again. It could be noticed that the length of each interval is different, because the distribution of the data in the universe is not uniform, and the clustering results to guide the division of subintervals can better reflect the data structure than the equally divided universe, which is beneficial to improve the prediction accuracy. Finally, the historical data is input into the corresponding interval so that each historical data corresponds to an interval [13].

Step 3: fuzzify the data and establish a logical relationship.

A fuzzy set $A_{i}$ is defined according to the interval obtained in the preceding step, and the historical data is fuzzified according to the following formula:

$$
\left\{\begin{array} { l } 
{ A _ { 1 } = \frac { f _ { 1 1 } } { u _ { 1 } } + \frac { f _ { 1 2 } } { u _ { 2 } } + \cdots + \frac { f _ { 1 n } } { u _ { n } } , } \\
{ A _ { 2 } = \frac { f _ { 2 1 } } { u _ { 1 } } + \frac { f _ { 2 2 } } { u _ { 2 } } + \cdots + \frac { f _ { 2 n } } { u _ { n } } , } \\
{ A _ { 3 } = \frac { f _ { 3 1 } } { u _ { 1 } } + \frac { f _ { 3 2 } } { u _ { 2 } } + \cdots + \frac { f _ { 3 n } } { u _ { n } } , } \\
{ \cdots } \\
{ A _ { n } = \frac { f _ { n 1 } } { u _ { 1 } } + \frac { f _ { n 2 } } { u _ { 2 } } + \cdots + \frac { f _ { n n } } { u _ { n } } , }
\end{array} \left\{\begin{array}{l}
A_{1}=\frac{1}{u_{1}}+\frac{0.5}{u_{2}}+\frac{0}{u_{3}}+\cdots+\frac{0}{u_{n}}, \\
A_{2}=\frac{0.5}{u_{1}}+\frac{1}{u_{2}}+\frac{0.5}{u_{3}}+\cdots+\frac{0}{u_{n}}, \\
A_{3}=\frac{0}{u_{1}}+\frac{0.5}{u_{2}}+\frac{1}{u_{3}}+\frac{0.5}{u_{4}}+\cdots+\frac{0}{u_{n}}, \\
\cdots \\
A_{n}=\frac{0}{u_{1}}+\frac{0}{u_{2}}+\cdots+\frac{0}{u_{n-1}}+\frac{1}{u_{n}} .
\end{array}\right.\right.
$$

After fuzzification, the fuzzy logic relationship is obtained according to Definition 3. For example, if year $n$ corresponds to $A_{i}$ and the year $n+1$ belongs to $A_{j}$, the logical relationship between them can be recorded as $A_{i} \longrightarrow A_{j}$.
Step 4: defuzzification and the prediction process. Based on the calculated defuzzification results, a dynamic prediction model of inbound tourism demand for ice and snow is constructed as presented in the following equation: 


$$
F_{j}=\frac{\left(P+m\left[A_{j}\right]\right)}{(S+1)} .
$$

\section{Output of Prediction Results Based on Improved Deep Belief Network}

4.1. Improved Deep Belief Network. Deep belief network (DBN) is a probabilistic generation model, a neural network with multiple hidden layers composed of multiple restricted Boltzmann machines. The Boltzmann machine named after Boltzmann distribution in statistical mechanics is popular because of its parallelism and adaptability to simple physical processes. Although unrestricted Boltzmann machine has very limited usefulness in practical or machine learning problem, the restricted Boltzmann machine (RBM) can learn efficiently within contained simple structure. The DBN is a combination of a generative model and the discriminant model. The generative model superimposes the layers of RBM so that the DBN model can extract features from the input data, layer by layer, to obtain high-level expressions. On the other hand, the discriminant model is a supervised neural network model used to complete the classification or prediction process. The improved deep belief network is developed on the basis of the traditional neural network while addressing its limitation regarding the depth of the hidden layer which cannot be expanded in traditional neural network. Conversely, the restricted Boltzmann machine is trained layer by layer through the gradient descent greedy algorithm while obtaining the interlayer weight " $\mathrm{W}$," the visible layer bias "c," and the hidden layer bias "b." In the training process, unsupervised learning is used primarily to extract the feature representation of the input data. On the basis of training stacked restricted Boltzmann machines, the backpropagation (BP) algorithm is used to fine-tune the system parameters [14].

In summary, the improved deep belief network can be regarded as a deep neural network composed of a multilayer unsupervised restricted Boltzmann machine network and a single-layer backpropagation neural network, as represented in Figure 1.

Since the improved deep belief network has strong learning ability, this paper selects the improved deep belief network to train the prediction model where the training of the deep belief network is conducted layer by layer; that is, after initializing the vector in the unit by mapping layer by layer from the first layer, the visible layer unit $v$ of the deep belief network is mapped to get the hidden layer unit $h$, and then the hidden layer unit $h$ will be used as the next visible layer which is mapped to get the hidden layer unit again and so on until the multilayer deep belief network is trained. During the training process, the weights of each layer are constantly updated according to the correlation between the hidden layer and the visible layer. The network reconstruction error is passed from the first layer of deep confidence network to the second layer and then to the third layer until it is propagated to the last layer of the deep confidence network [15].

4.2. Predictive Model Learning and Training Based on Improved Deep Belief Network. Since the improved deep belief network is trained layer by layer, it cannot guarantee that the feature vector of the entire network layer will be optimally mapped after training; it can only ensure the optimal weight adjustments in the respective layers; that is, the nonlinear mapping of the feature vector in the particular layer is optimal. Therefore, the output data of the deep belief network is entered into the BP neural network for model learning, and the model parameters $\theta=(W, b, c)$ and learning rate $\varepsilon$ after pretraining are obtained. The calculation formula of the error gradient $\sigma_{i}$ of all visible layer units $v_{i}$ is then calculated as

$$
\sigma_{i}=Q_{i}\left(1-Q_{i}\right)\left(e_{i}-Q_{i}\right),
$$

where $e_{i}$ represents the desired output and $Q_{i}$ represents the output unit of the visible layer. Subsequently, the calculation formula of the error gradient $\sigma_{j}$ of all hidden layer units $h_{i}$ is given by

$$
\sigma_{j}=Q_{i}\left(1-Q_{i}\right) \sum_{i} \theta_{i j} \sigma_{i} h_{i}
$$

Using the error gradient value, the dynamic forecast error function of ice and snow inbound tourism demand is constructed as per the following equation:

$$
E_{t}=\sigma_{j}+(1-\gamma) g_{t},
$$

where $g_{t}$ represents the gradient at time $t$ and $\gamma$ represents the expected value coefficient.

In the end, the dynamic forecasting model of ice and snow inbound tourism demand is given the input data to train the model and as per the error function, test data is tested on the trained model to output the dynamic prediction value of ice and snow inbound tourism demand in the output layer $P$ as given in the following equation:

$$
P_{P}=E_{t} C H \text {, }
$$

where $C$ represents the state of the deep confidence network memory module and $H$ represents the output of the memory module.

\section{Simulation Experiment Analysis}

In order to verify the effectiveness of the dynamic prediction algorithm of inbound tourism demand for ice and snow-based on the improved deep confidence network proposed in this paper in practical application, a simulation experiment analysis is carried out and to ensure the accuracy and fairness of the experiment, the requirements for ice and snow inbound tourism were entered into the database in advance. The database data used by the experimental group and the comparison group are exactly the same. The equipment hardware environment is shown in Table 1.

For an effective analysis, the proposed method in the current study is compared with the tourism demand forecasting algorithm based on the improved grey model proposed by Yao et al. [4] and the improved fruit fly method proposed by Mingyang et. al. [5] which optimized the travel demand prediction of the echo state network. The comparison results are presented in Figure 2. 


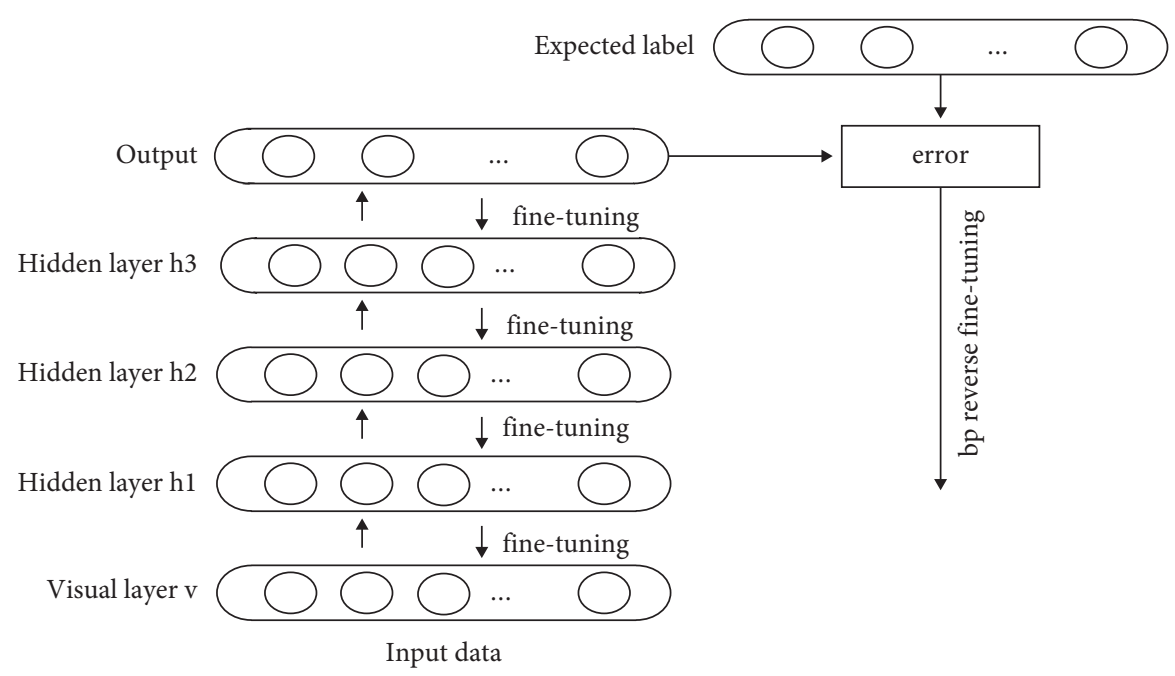

FIGURE 1: Improved deep belief network.

TABLE 1: Hardware parameter table.

\begin{tabular}{lc}
\hline Experimental environment & Parameter \\
\hline Software & Hadoop \\
Processor & Intel Xeon ES-2630 v2 \\
RAM & 16 GB \\
Storage disk & 50 TB \\
Network & Gigabit Ethernet card \\
Operating system & eServer 11 SP2 64 bit \\
\hline
\end{tabular}

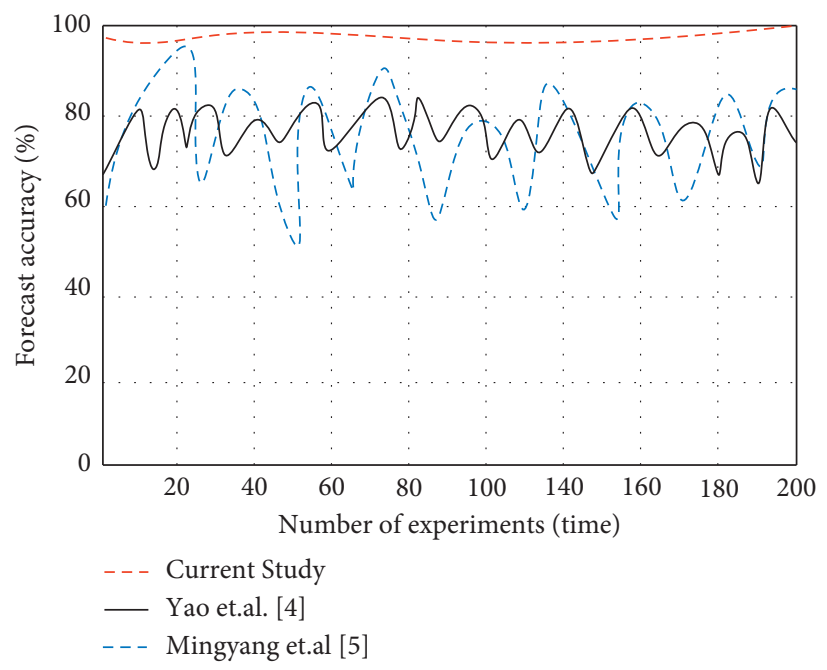

FIgURE 2: Comparison results of dynamic prediction accuracy of inbound tourism demand for ice and snow.

According to Figure 2, the dynamic prediction algorithm based on the improved deep confidence network proposed in this paper is more than $95 \%$ accurate which is better than both the previously proposed methods.

To further verify the effectiveness of the proposed technique, the total prediction time taken by the proposed study is mapped against the methods of Yao et al. [4] and
Mingyang et. al. [5], and the results are presented in Figure 3.

According to Figure 3, it can be noticed that the prediction time of the current study based on the improved deep confidence network is within 20 seconds, which is significantly shorter than the prediction time of the existing techniques. 


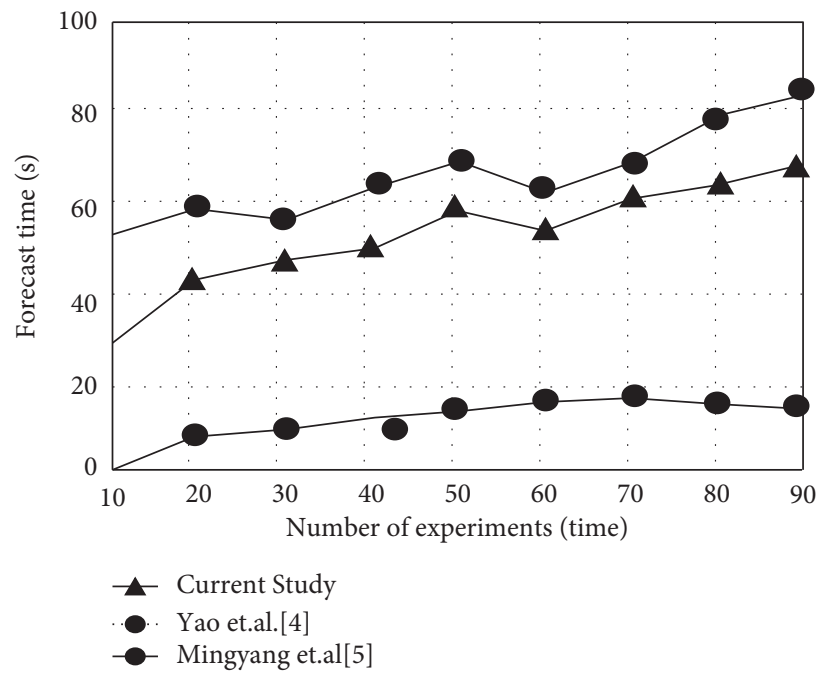

FIgURE 3: Comparison results of the dynamic forecast time of inbound tourism demand for ice and snow.

\section{Conclusion}

Under the background of the era of stimulating future economic growth with consumption, the tourism industry is facing fundamental changes with increased international influence, upgradation of strategic functions, strengthening of basic functions, and prominent status of the main body. The accurate forecast of the number of tourists visiting a particular area at a certain time of the year can be used by the local government to promote tourism in a better way by providing an improved tourists experience which will contribute to the overall economic development of the area. It will also help in providing relevant technical support for formulating tourism development policies and guiding the rational use and allocation of tourism market resources. Considering these arguments, this paper proposed a dynamic prediction algorithm for inbound tourism demand based on fuzzy time series and improved deep confidence network. Simulation experiments verified the effectiveness and practicability of this method testifying that the algorithm can more accurately predict the demand for inbound tourism in ice and snow as compared to its preceding counterparts. As such, this will contribute to providing better services to the inbound tourists and will positively elicit the tourism industry in the region, thereby earning better revenue for the local people and the region. Moreover, it is encouraged to utilize the same study for other tourismrelated predictions which may prove its generalizability and will help in its adoption for real-world solutions.

\section{Data Availability}

The data used to support the findings of this study are available from the corresponding author upon request.

\section{Conflicts of Interest}

The authors declare that they have no known competing financial interests or personal relationships that could have appeared to influence the work reported in this study.

\section{Acknowledgments}

This study was supported by Qiqihar City Philosophy and Social Science Research Planning Project: Research on the status and development PATH of Qiqihar city's ice and snow sports industry under the background of the Beijing Winter Olympics (QSX2020-30YB).

\section{References}

[1] W. Jun, L. Yuyan, T. Lingyu, and G. Peng, "Modeling a combined forecast algorithm based on sequence patterns and near characteristics: an application for tourism demand forecasting," Chaos, Solitons \& Fractals, vol. 108, pp. 136-147, 2018.

[2] Y. Wang and P. Zhang, "Research on demand forecast of entry tourism in chengdu based on combination of various models," Mathematics practice and knowledge, vol. 49, no. 10, pp. 299-302, 2019.

[3] Y. Xu, F. Jiang, J. Du, and D. Gong, "A cross-domain collaborative filtering algorithm with expanding user and item features via the latent factor space of auxiliary domains," Pattern Recognition, vol. 94, pp. 96-109, 2019.

[4] L. Yao, C. Han, and J. Ma, "Research on tourism demand forecast based on improved grey model," Computer Science, vol. 45, no. 1, pp. 122-127, 2018.

[5] Y. Xu, J. Yang, and Z. Xie, "Training SVMs on a bound vectors set based on Fisher projection," Frontiers of Computer Science, vol. 8, no. 5, pp. 793-806, 2014.

[6] Z. Liang and Y. Kuang, "Research on autoregressive distribution and lag model forecasting of tourism demand based on searching big data," Productivity research, vol. 0, no. 2, pp. 15-22, 2018.

[7] Z. Han and Z. Fang, "Application of GIOWHA-GALSSVRSARIMA combined model in tourism demand forecasting," Mathematics practice and knowledge, vol. 49, no. 19, pp. 6979, 2019.

[8] Y. Xiao, X. Tian, and J. J. Liu, "Tourism traffic demand prediction using google trends based on EEMD-DBN," Engineering, vol. 12, no. 3, pp. 194-215, 2020.

[9] C. Colther and A. Arriagada-Millaman, "Pronóstico de la demanda turística de Chile basado en modelos lineales y no 
lineales estacionales," PASOS : Revista de Turismo y Patrimonio Cultural, vol. 19, no. 2, pp. 323-336, 2021.

[10] X. Jiao, G. Li, and J. L. Chen, "Forecasting international tourism demand: a local spatiotemporal model," Annals of Tourism Research, vol. 83, p. 102937, 2020.

[11] C. Zhang, S. Wang, S. Sun, and Y. Wei, "Knowledge mapping of tourism demand forecasting research," Tourism Management Perspectives, vol. 35, p. 100715, 2020.

[12] W. Wang, "Application of the optimal linear combination forecast model in tourism demand forecasting: a case of forecasting of visitors from mainland China to Macao," Mathematics practice and knowledge, vol. 48, no. 12, pp. 49-58, 2018.

[13] E. S. Silva, H. Hassani, S. Heravi, and X. Huang, "Forecasting tourism demand with denoised neural networks," Annals of Tourism Research, vol. 74, pp. 134-154, 2019.

[14] M. Yu, T. Quan, Q. Peng, X. Yu, and L. Liu, "A model-based collaborate filtering algorithm based on stacked AutoEncoder," Neural Computing \& Applications, 2021

[15] H. Zhang and Y. Xu, "Real-time tracking and forecasting simulation of tourist flow data in scenic spots under cloud computing," Computer Simulation, vol. 36, no. 10, pp. 467$471,2019$. 